\title{
Glycosylation of Serum Clusterin in Wild-Type Transthyretin-Associated (ATTRwt) Amyloidosis: A study of Disease-Associated Compositional Features Using Mass Spectrometry Analyses
}

Celia M. Torres-Arancivia, ${ }^{\perp}$ Deborah Chang, ${ }^{\dagger}$ William E. Hackett, ${ }^{\dagger}$ Joseph Zaia, ${ }^{\dagger}$ and Lawreen H. Connors $* \perp \S$.

${ }^{\perp}$ Amyloidosis Center, ${ }^{\dagger}$ Center for Biomedical Mass Spectrometry, ${ }^{\S}$ Department of Pathology and Laboratory Medicine, Boston University School of Medicine, Boston, MA 02118, USA 
Supporting Information

\begin{tabular}{|c|c|c|c|c|c|c|c|c|c|c|c|c|}
\hline & \multicolumn{6}{|c|}{ ATTRwt CLUSTERIN } & \multicolumn{6}{|c|}{ CONTROL CLUSTERIN } \\
\hline $\mathrm{m} / \mathrm{z}$ & No & Glycan & $\begin{array}{l}\text { Average } \\
\text { retention } \\
\text { time }\end{array}$ & $\begin{array}{l}\text { No. of } \\
\text { observations } \\
\text { per replicate }\end{array}$ & $\begin{array}{l}\text { Charge } \\
\text { states } \\
\text { per } \\
\text { replicate } \\
\end{array}$ & $\begin{array}{l}\text { Average } \\
\text { mass } \\
\text { error }\end{array}$ & No & Glycan & $\begin{array}{l}\text { Average } \\
\text { retention } \\
\text { time }\end{array}$ & $\begin{array}{c}\text { No. of } \\
\text { observations } \\
\text { per replicate }\end{array}$ & $\begin{array}{l}\text { Charge } \\
\text { states } \\
\text { per } \\
\text { replicate } \\
\end{array}$ & $\begin{array}{c}\text { Average } \\
\text { mass } \\
\text { error }\end{array}$ \\
\hline \multicolumn{13}{|c|}{ EDALN*ETR $(\alpha 64 N)$} \\
\hline \multirow[t]{4}{*}{3151.2} & 1 & {$[4 ; 5 ; 0 ; 2]$} & 19.4 & $18,1,16,2$ & $3,2,3,3$ & $4.48 \mathrm{E}-03$ & 1 & {$[4 ; 5 ; 0 ; 2]$} & 19.3 & $13,13,14,7$ & $3,3,3,3$ & $1.56 \mathrm{E}-03$ \\
\hline & & {$[4 ; 5 ; 0 ; 2]$} & 20.0 & $1,16,1,1$ & $4,3,4,2$ & $3.03 \mathrm{E}-03$ & & {$[4 ; 5 ; 0 ; 2]$} & 19.5 & $0,0,5,0$ & $0,0,4,0$ & 4.07E-04 \\
\hline & & {$[4 ; 5 ; 0 ; 2]$} & 20.2 & $0,2,0,17$ & $0,4,0,3$ & $8.35 \mathrm{E}-04$ & & & & & & \\
\hline & & {$[4 ; 5 ; 0 ; 2]$} & 20.4 & $0,0,0,7$ & $0,0,0,4$ & $6.62 \mathrm{E}-04$ & & & & & & \\
\hline 3006.2 & & & & & & & 2 & {$[4 ; 5 ; 1 ; 1]$} & 18.0 & $1,0,0,0$ & $3,0,0,0$ & $1.33 \mathrm{E}-03$ \\
\hline 3297.3 & 2 & {$[4 ; 5 ; 1 ; 2]$} & 20.5 & $5,5,3,4$ & $3,3,3,3$ & $1.48 \mathrm{E}-03$ & 3 & {$[4 ; 5 ; 1 ; 2]$} & 20.1 & $3,2,4,2$ & $3,3,3,3$ & $2.33 \mathrm{E}-03$ \\
\hline 3152.2 & & & & & & & 4 & {$[4 ; 5 ; 2 ; 1]$} & 18.8 & $1,0,1,0$ & $3,0,3,0$ & $1.68 \mathrm{E}-02$ \\
\hline 3354.3 & 3 & {$[5 ; 5 ; 0 ; 2]$} & 20.6 & $1,0,2,0$ & $3,0,3,0$ & $2.01 \mathrm{E}-03$ & 5 & {$[5 ; 5 ; 0 ; 2]$} & 21.0 & $2,2,0,0$ & $3,3,0,0$ & $2.24 \mathrm{E}-03$ \\
\hline 3209.2 & 4 & {$[5 ; 5 ; 1 ; 1]$} & 18.3 & $0,1,0,0$ & $0,3,0,0$ & $1.52 \mathrm{E}-02$ & & & & & & \\
\hline \multirow[t]{2}{*}{3516.3} & 5 & {$[5 ; 6 ; 0 ; 2]$} & 19.7 & $4,4,4,4$ & $3,3,3,3$ & $1.72 \mathrm{E}-03$ & 6 & {$[5 ; 6 ; 0 ; 2]$} & 19.3 & $3,4,4,2$ & $3,3,3,3$ & $4.30 \mathrm{E}-03$ \\
\hline & & {$[5 ; 6 ; 0 ; 2]$} & 19.7 & $1,0,1,2$ & $4,0,4,4$ & $3.79 \mathrm{E}-03$ & & {$[5 ; 6 ; 0 ; 2]$} & 19.5 & $2,0,3,0$ & $4,0,4,0$ & $1.30 \mathrm{E}-03$ \\
\hline \multirow[t]{2}{*}{3807.4} & 6 & {$[5 ; 6 ; 0 ; 3]$} & 23.1 & $22,24,22,15$ & $3,3,3,3$ & $1.95 \mathrm{E}-03$ & 7 & {$[5 ; 6 ; 0 ; 3]$} & 22.5 & $23,22,20,18$ & $3,3,3,3$ & $2.00 \mathrm{E}-03$ \\
\hline & & {$[5 ; 6 ; 0 ; 3]$} & 23.1 & $20,18,21,22$ & $4,4,4,4$ & 7.04E-04 & & {$[5 ; 6 ; 0 ; 3]$} & 22.5 & $22,18,25,22$ & $4,4,4,4$ & 8.77E-04 \\
\hline \multirow[t]{2}{*}{3662.4} & 7 & {$[5 ; 6 ; 1 ; 2]$} & 19.6 & $5,3,3,2$ & $3,3,3,3$ & 4.19E-03 & 8 & {$[5 ; 6 ; 1 ; 2]$} & 19.3 & $4,3,2,0$ & $3,3,3,0$ & $1.44 \mathrm{E}-03$ \\
\hline & & {$[5 ; 6 ; 1 ; 2]$} & 19.6 & $1,1,1,2$ & $4,4,4,4$ & $4.88 \mathrm{E}-03$ & & {$[5 ; 6 ; 1 ; 2]$} & 19.4 & $1,0,3,0$ & $4,0,4,0$ & $4.51 \mathrm{E}-03$ \\
\hline \multirow[t]{2}{*}{3953.5} & 8 & {$[5 ; 6 ; 1 ; 3]$} & 22.9 & $19,19,18,12$ & $3,3,3,3$ & $3.32 \mathrm{E}-03$ & 9 & {$[5 ; 6 ; 1 ; 3]$} & 22.2 & $16,15,13,14$ & $3,3,3,3$ & $3.01 \mathrm{E}-03$ \\
\hline & & {$[5 ; 6 ; 1 ; 3]$} & 23.0 & $17,19,20,22$ & $4,4,4,4$ & $1.50 \mathrm{E}-03$ & & {$[5 ; 6 ; 1 ; 3]$} & 22.2 & $18,13,22,15$ & $4,4,4,4$ & $1.63 \mathrm{E}-03$ \\
\hline 3808.5 & 9 & {$[5 ; 6 ; 2 ; 2]$} & 20.2 & $0,0,1,0$ & $0,0,4,0$ & $4.36 \mathrm{E}-03$ & 10 & {$[5 ; 6 ; 2 ; 2]$} & 21.3 & $0,1,0,0$ & $0,4,0,0$ & $2.94 \mathrm{E}-02$ \\
\hline 4099.6 & 10 & {$[5 ; 6 ; 2 ; 3]$} & 22.9 & $2,2,1,2$ & $3,3,3,4$ & $5.59 \mathrm{E}-03$ & 11 & {$[5 ; 6 ; 2 ; 3]$} & 22.6 & $0,1,2,0$ & $0,4,4,0$ & $1.32 \mathrm{E}-02$ \\
\hline
\end{tabular}




\begin{tabular}{|c|c|c|c|c|c|c|c|c|c|c|c|c|}
\hline & & {$[5 ; 6 ; 2 ; 3]$} & 22.8 & $2,2,3,0$ & $4,4,4,0$ & $4.31 \mathrm{E}-03$ & & & & & & \\
\hline \multirow[t]{2}{*}{3954.5} & 11 & {$[5 ; 6 ; 3 ; 2]$} & 21.3 & $2,1,1,0$ & $4,3,4,0$ & $1.47 \mathrm{E}-02$ & 12 & {$[5 ; 6 ; 3 ; 2]$} & 21.0 & $0,1,0,0$ & $0,4,0,0$ & $1.53 \mathrm{E}-02$ \\
\hline & & {$[5 ; 6 ; 3 ; 2]$} & 20.4 & $0,1,0,0$ & $0,4,0,0$ & $2.11 \mathrm{E}-02$ & & & & & & \\
\hline \multirow[t]{2}{*}{3824.5} & 12 & {$[5 ; 7 ; 1 ; 2]$} & 23.2 & $3,7,3,3$ & $3,3,3,3$ & $1.35 \mathrm{E}-02$ & 13 & {$[5 ; 7 ; 1 ; 2]$} & 22.4 & $6,6,2,5$ & $3,3,3,3$ & $1.32 \mathrm{E}-02$ \\
\hline & & {$[5 ; 7 ; 1 ; 2]$} & 23.1 & $2,3,5,3$ & $4,4,4,4$ & $1.29 \mathrm{E}-02$ & & {$[5 ; 7 ; 1 ; 2]$} & 22.4 & $2,2,3,4$ & $4,4,4,4$ & $1.39 \mathrm{E}-02$ \\
\hline \multirow[t]{2}{*}{3970.5} & 13 & {$[5 ; 7 ; 2 ; 2]$} & 22.9 & $7,8,3,2$ & $3,3,3,3$ & $1.24 \mathrm{E}-02$ & 14 & {$[5 ; 7 ; 2 ; 2]$} & 22.2 & $5,3,1,4$ & $3,3,3,3$ & $1.19 \mathrm{E}-02$ \\
\hline & & {$[5 ; 7 ; 2 ; 2]$} & 22.9 & $3,4,7,4$ & $4,4,4,4$ & $1.29 \mathrm{E}-02$ & & {$[5 ; 7 ; 2 ; 2]$} & 22.2 & $2,4,5,6$ & $4,4,4,4$ & $1.25 \mathrm{E}-02$ \\
\hline \multirow[t]{2}{*}{3825.5} & 14 & {$[5 ; 7 ; 3 ; 1]$} & 22.4 & $0,1,0,0$ & $0,3,0,0$ & 8.32E-03 & 15 & {$[5 ; 7 ; 3 ; 1]$} & 23.8 & $1,0,0,0$ & $3,0,0,0$ & $2.94 \mathrm{E}-03$ \\
\hline & & & & & & & & {$[5 ; 7 ; 3 ; 1]$} & 23.6 & $1,0,0,0$ & $4,0,0,0$ & $1.54 \mathrm{E}-02$ \\
\hline 3977.5 & & & & & & & 16 & {$[6 ; 4 ; 0 ; 4]$} & 23.6 & $1,0,0,0$ & $4,0,0,0$ & $1.99 \mathrm{E}-02$ \\
\hline 3978.5 & & & & & & & 17 & {$[6 ; 4 ; 2 ; 3]$} & 23.5 & $1,0,0,0$ & $4,0,0,0$ & $2.63 \mathrm{E}-02$ \\
\hline \multirow[t]{2}{*}{4010.5} & 15 & {$[6 ; 6 ; 0 ; 3]$} & 23.3 & $8,8,7,5$ & $3,3,3,3$ & $3.81 \mathrm{E}-03$ & 18 & {$[6 ; 6 ; 0 ; 3]$} & 22.6 & $7,7,6,6$ & $3,3,3,3$ & $3.67 \mathrm{E}-03$ \\
\hline & & {$[6 ; 6 ; 0 ; 3]$} & 23.3 & $10,8,8,10$ & $4,4,4,4$ & $3.25 \mathrm{E}-03$ & & {$[6 ; 6 ; 0 ; 3]$} & 22.6 & $10,6,12,8$ & $4,4,4,4$ & $2.53 \mathrm{E}-03$ \\
\hline 3720.4 & & & & & & & 19 & {$[6 ; 6 ; 2 ; 1]$} & 20.3 & $1,0,0,0$ & $3,0,0,0$ & $8.01 \mathrm{E}-03$ \\
\hline \multicolumn{13}{|c|}{ KEDALN*ETR $(\alpha 64 N)$} \\
\hline 3426.4 & 16 & {$[4 ; 5 ; 3 ; 1]$} & 18.5 & $0,1,0,0$ & $0,3,0,0$ & $1.08 \mathrm{E}-02$ & & & & & & \\
\hline \multirow[t]{2}{*}{3935.5} & 17 & {$[5 ; 6 ; 0 ; 3]$} & 18.4 & $7,6,6,4$ & $3,3,3,3$ & $1.55 \mathrm{E}-03$ & 20 & {$[5 ; 6 ; 0 ; 3]$} & 17.9 & $6,5,5,1$ & $3,3,3,3$ & $1.53 \mathrm{E}-03$ \\
\hline & & {$[5 ; 6 ; 0 ; 3]$} & 18.4 & $6,6,7,6$ & $4,4,4,4$ & $6.17 \mathrm{E}-04$ & & {$[5 ; 6 ; 0 ; 3]$} & 17.9 & $8,6,7,2$ & $4,4,4,4$ & $1.93 \mathrm{E}-03$ \\
\hline \multirow[t]{2}{*}{4081.6} & 18 & {$[5 ; 6 ; 1 ; 3]$} & 18.0 & $5,6,4,1$ & $3,3,3,3$ & $3.85 \mathrm{E}-03$ & 21 & {$[5 ; 6 ; 1 ; 3]$} & 17.7 & $4,3,1,1$ & $3,3,3,4$ & $3.23 \mathrm{E}-03$ \\
\hline & & {$[5 ; 6 ; 1 ; 3]$} & 18.1 & $8,7,7,6$ & $4,4,4,4$ & $1.72 \mathrm{E}-03$ & & {$[5 ; 6 ; 1 ; 3]$} & 17.8 & $4,4,7,0$ & $4,4,4,0$ & $2.63 \mathrm{E}-03$ \\
\hline 3936.6 & & & & & & & 22 & {$[5 ; 6 ; 2 ; 2]$} & 17.4 & $0,0,1,0$ & $0,0,4,0$ & $1.58 \mathrm{E}-02$ \\
\hline 4227.6 & 19 & {$[5 ; 6 ; 2 ; 3]$} & 18.0 & $0,1,1,0$ & $0,4,4,0$ & 4.11E-03 & & & & & & \\
\hline \multirow[t]{2}{*}{3952.5} & 20 & {$[5 ; 7 ; 1 ; 2]$} & 18.2 & $1,0,1,0$ & $3,0,4,0$ & $1.57 \mathrm{E}-02$ & 23 & {$[5 ; 7 ; 1 ; 2]$} & 18.1 & $1,0,1,0$ & $4,0,4,0$ & $5.24 \mathrm{E}-03$ \\
\hline & & {$[5 ; 7 ; 1 ; 2]$} & 18.4 & $1,0,0,0$ & $4,0,0,0$ & $1.29 \mathrm{E}-02$ & & & & & & \\
\hline 4105.6 & 21 & {$[6 ; 4 ; 0 ; 4]$} & 18.6 & $2,0,1,1$ & $4,0,4,4$ & $1.69 \mathrm{E}-02$ & 24 & {$[6 ; 4 ; 0 ; 4]$} & 18.1 & $0,0,1,0$ & $0,0,4,0$ & 7.35E-03 \\
\hline \multirow[t]{2}{*}{4106.6} & 22 & {$[6 ; 4 ; 2 ; 3]$} & 19.0 & $0,0,0,1$ & $0,0,0,4$ & $2.50 \mathrm{E}-02$ & 25 & {$[6 ; 4 ; 2 ; 3]$} & 18.1 & $4,1,2,1$ & $4,3,4,4$ & $2.31 \mathrm{E}-02$ \\
\hline & & & & & & & & {$[6 ; 4 ; 2 ; 3]$} & 18.6 & $0,4,0,0$ & $0,4,0,0$ & $2.29 \mathrm{E}-02$ \\
\hline \multirow[t]{2}{*}{4138.6} & 23 & {$[6 ; 6 ; 0 ; 3]$} & 18.1 & $4,3,3,5$ & $3,3,3,4$ & $2.52 \mathrm{E}-03$ & 26 & {$[6 ; 6 ; 0 ; 3]$} & 17.7 & $2,3,2,1$ & $3,3,3,4$ & $1.51 \mathrm{E}-03$ \\
\hline & & {$[6 ; 6 ; 0 ; 3]$} & 17.9 & $8,6,6,0$ & $4,4,4,0$ & 5.89E-04 & & {$[6 ; 6 ; 0 ; 3]$} & 17.8 & $7,6,8,0$ & $4,4,4,0$ & $1.71 \mathrm{E}-03$ \\
\hline
\end{tabular}




\begin{tabular}{|c|c|c|c|c|c|c|c|c|c|c|c|c|}
\hline 4284.7 & 24 & {$[6 ; 6 ; 1 ; 3]$} & 18.2 & $1,0,0,0$ & $4,0,0,0$ & $1.35 \mathrm{E}-03$ & & & & & & \\
\hline \multicolumn{13}{|c|}{ KKEDALN*ETR $(\alpha 64 N)$} \\
\hline 3432.4 & & & & & & & 27 & {$[3 ; 10 ; 0 ; 0]$} & 16.3 & $1,0,0,0$ & $4,0,0,0$ & $1.34 \mathrm{E}-02$ \\
\hline 3116.3 & 25 & {$[4 ; 5 ; 0 ; 1]$} & 13.3 & $1,0,1,2$ & $3,0,4,4$ & $1.33 \mathrm{E}-03$ & 28 & {$[4 ; 5 ; 0 ; 1]$} & 13.1 & $2,0,0,0$ & $4,0,0,0$ & $4.25 \mathrm{E}-03$ \\
\hline \multirow[t]{2}{*}{3407.4} & 26 & {$[4 ; 5 ; 0 ; 2]$} & 14.2 & $3,5,6,7$ & $3,3,3,3$ & $5.04 \mathrm{E}-03$ & 29 & {$[4 ; 5 ; 0 ; 2]$} & 13.7 & $4,3,3,1$ & $3,3,4,3$ & $6.43 \mathrm{E}-03$ \\
\hline & & {$[4 ; 5 ; 0 ; 2]$} & 14.2 & $5,5,5,11$ & $4,4,4,4$ & $2.12 \mathrm{E}-03$ & & {$[4 ; 5 ; 0 ; 2]$} & 13.7 & $8,5,0,4$ & $4,4,0,4$ & $3.87 \mathrm{E}-03$ \\
\hline \multirow[t]{2}{*}{3553.5} & 27 & {$[4 ; 5 ; 1 ; 2]$} & 14.9 & $1,1,3,2$ & $3,3,4,4$ & 5.84E-03 & 30 & {$[4 ; 5 ; 1 ; 2]$} & 14.3 & $2,2,0,1$ & $3,4,0,3$ & $1.82 \mathrm{E}-03$ \\
\hline & & {$[4 ; 5 ; 1 ; 2]$} & 14.5 & $3,2,0,0$ & $4,4,0,0$ & $4.02 \mathrm{E}-03$ & & {$[4 ; 5 ; 1 ; 2]$} & 14.0 & $4,0,0,1$ & $4,0,0,4$ & $1.36 \mathrm{E}-03$ \\
\hline 3432.4 & & & & & & & 31 & {$[5 ; 3 ; 1 ; 2]$} & 16.3 & $1,0,0,0$ & $4,0,0,0$ & $2.43 \mathrm{E}-02$ \\
\hline \multirow[t]{2}{*}{3610.5} & 28 & {$[5 ; 5 ; 0 ; 2]$} & 14.8 & $1,1,1,1$ & $3,3,3,4$ & $1.99 \mathrm{E}-03$ & 32 & {$[5 ; 5 ; 0 ; 2]$} & 14.2 & $4,1,3,1$ & $3,3,4,3$ & $2.98 \mathrm{E}-03$ \\
\hline & & {$[5 ; 5 ; 0 ; 2]$} & 14.6 & $3,1,2,0$ & $4,4,4,0$ & $2.31 \mathrm{E}-03$ & & {$[5 ; 5 ; 0 ; 2]$} & 14.2 & $6,2,0,3$ & $4,4,0,4$ & $2.34 \mathrm{E}-03$ \\
\hline \multirow[t]{2}{*}{3772.5} & 29 & {$[5 ; 6 ; 0 ; 2]$} & 14.6 & $1,1,1,2$ & $3,3,3,4$ & $2.41 \mathrm{E}-03$ & 33 & {$[5 ; 6 ; 0 ; 2]$} & 13.9 & $3,2,4,1$ & $3,4,4,3$ & $2.58 \mathrm{E}-03$ \\
\hline & & {$[5 ; 6 ; 0 ; 2]$} & 14.1 & $6,4,4,0$ & $4,4,4,0$ & $1.79 \mathrm{E}-03$ & & {$[5 ; 6 ; 0 ; 2]$} & 13.5 & $7,0,0,4$ & $4,0,0,4$ & $6.56 \mathrm{E}-03$ \\
\hline \multirow[t]{3}{*}{4063.6} & 30 & {$[5 ; 6 ; 0 ; 3]$} & 15.6 & $6,3,2,1$ & $3,3,3,3$ & $1.84 \mathrm{E}-03$ & 34 & {$[5 ; 6 ; 0 ; 3]$} & 15.0 & $6,2,2,4$ & $3,3,3,3$ & $3.41 \mathrm{E}-03$ \\
\hline & & {$[5 ; 6 ; 0 ; 3]$} & 15.6 & $13,10,12,11$ & $4,4,4,4$ & $2.30 \mathrm{E}-03$ & & {$[5 ; 6 ; 0 ; 3]$} & 15.0 & $11,10,10,10$ & $4,4,4,4$ & $3.76 \mathrm{E}-03$ \\
\hline & & {$[5 ; 6 ; 0 ; 3]$} & 16.1 & $0,0,0,1$ & $0,0,0,5$ & $3.81 \mathrm{E}-03$ & & {$[5 ; 6 ; 0 ; 3]$} & 14.1 & $0,0,0,2$ & $0,0,0,5$ & $6.86 \mathrm{E}-03$ \\
\hline \multirow[t]{2}{*}{3918.6} & 31 & {$[5 ; 6 ; 1 ; 2]$} & 14.1 & $4,2,2,5$ & $4,4,4,4$ & $3.18 \mathrm{E}-03$ & 35 & {$[5 ; 6 ; 1 ; 2]$} & 13.8 & $1,0,0,0$ & $3,0,0,0$ & $3.09 \mathrm{E}-02$ \\
\hline & & & & & & & & {$[5 ; 6 ; 1 ; 2]$} & 13.9 & $2,0,0,0$ & $4,0,0,0$ & $1.78 \mathrm{E}-02$ \\
\hline \multirow[t]{3}{*}{4209.7} & 32 & {$[5 ; 6 ; 1 ; 3]$} & 15.4 & $5,3,2,8$ & $3,3,3,4$ & $2.55 \mathrm{E}-03$ & 36 & {$[5 ; 6 ; 1 ; 3]$} & 14.8 & $4,1,1,3$ & $3,3,3,3$ & $3.73 \mathrm{E}-03$ \\
\hline & & {$[5 ; 6 ; 1 ; 3]$} & 15.4 & $13,10,8,1$ & $4,4,4,5$ & $2.23 \mathrm{E}-03$ & & {$[5 ; 6 ; 1 ; 3]$} & 14.8 & $12,7,6,6$ & $4,4,4,4$ & $1.55 \mathrm{E}-03$ \\
\hline & & {$[5 ; 6 ; 1 ; 3]$} & 14.9 & $0,0,1,0$ & $0,0,5,0$ & $2.85 \mathrm{E}-04$ & & {$[5 ; 6 ; 1 ; 3]$} & 14.7 & $2,0,1,1$ & $5,0,5,5$ & $2.22 \mathrm{E}-03$ \\
\hline 4064.6 & 33 & {$[5 ; 6 ; 2 ; 2]$} & 15.5 & $1,0,0,0$ & $5,0,0,0$ & $7.75 \mathrm{E}-03$ & & & & & & \\
\hline 4355.7 & 34 & {$[5 ; 6 ; 2 ; 3]$} & 15.4 & $2,3,2,2$ & $4,4,4,4$ & $2.76 \mathrm{E}-03$ & 37 & {$[5 ; 6 ; 2 ; 3]$} & 14.5 & $1,0,0,1$ & $4,0,0,4$ & $2.36 \mathrm{E}-02$ \\
\hline \multirow[t]{2}{*}{4210.7} & & & & & & & 38 & {$[5 ; 6 ; 3 ; 2]$} & 16.0 & $0,0,1,0$ & $0,0,4,0$ & $1.41 \mathrm{E}-02$ \\
\hline & 35 & {$[5 ; 7 ; 1 ; 2]$} & 15.7 & $1,0,0,0$ & $4,0,0,0$ & $1.69 \mathrm{E}-02$ & & & & & & \\
\hline \multirow[t]{2}{*}{4233.7} & 36 & {$[6 ; 4 ; 0 ; 4]$} & 15.7 & $1,1,1,2$ & $4,4,4,4$ & 8.03E-03 & 39 & {$[6 ; 4 ; 0 ; 4]$} & 15.0 & $7,3,4,4$ & $4,4,4,4$ & 7.99E-03 \\
\hline & & & & & & & & {$[6 ; 4 ; 0 ; 4]$} & 14.8 & $0,0,1,0$ & $0,0,5,0$ & $1.99 \mathrm{E}-02$ \\
\hline \multirow[t]{2}{*}{4234.7} & 37 & {$[6 ; 4 ; 2 ; 3]$} & 15.9 & $6,8,6,6$ & $4,4,4,4$ & $2.68 \mathrm{E}-02$ & 40 & {$[6 ; 4 ; 2 ; 3]$} & 15.3 & $4,11,11,2$ & $3,4,4,3$ & $2.70 \mathrm{E}-02$ \\
\hline & & & & & & & & {$[6 ; 4 ; 2 ; 3]$} & 15.1 & $9,0,0,13$ & $4,0,0,4$ & $2.44 \mathrm{E}-02$ \\
\hline
\end{tabular}




\begin{tabular}{|c|c|c|c|c|c|c|c|c|c|c|c|c|}
\hline & & & & & & & & {$[6 ; 4 ; 2 ; 3]$} & 14.8 & $0,0,0,1$ & $0,0,0,5$ & $2.40 \mathrm{E}-02$ \\
\hline \multirow[t]{2}{*}{3975.6} & 38 & {$[6 ; 6 ; 0 ; 2]$} & 14.1 & $3,4,1,4$ & $4,4,4,4$ & $5.94 \mathrm{E}-03$ & 41 & {$[6 ; 6 ; 0 ; 2]$} & 13.5 & $1,2,1,3$ & $3,4,4,4$ & $5.40 \mathrm{E}-03$ \\
\hline & & & & & & & & {$[6 ; 6 ; 0 ; 2]$} & 13.8 & $6,0,0,0$ & $4,0,0,0$ & $1.32 \mathrm{E}-02$ \\
\hline \multirow[t]{3}{*}{4266.7} & 39 & {$[6 ; 6 ; 0 ; 3]$} & 15.3 & $4,4,2,13$ & $3,3,3,4$ & $9.30 \mathrm{E}-04$ & 42 & {$[6 ; 6 ; 0 ; 3]$} & 14.8 & $5,3,2,6$ & $3,3,3,3$ & $2.06 \mathrm{E}-03$ \\
\hline & & {$[6 ; 6 ; 0 ; 3]$} & 15.3 & $14,13,13,1$ & $4,4,4,5$ & $2.89 \mathrm{E}-03$ & & {$[6 ; 6 ; 0 ; 3]$} & 14.8 & $12,9,10,11$ & $4,4,4,4$ & $1.62 \mathrm{E}-03$ \\
\hline & & {$[6 ; 6 ; 0 ; 3]$} & 14.9 & $0,0,1,0$ & $0,0,5,0$ & $1.04 \mathrm{E}-02$ & & {$[6 ; 6 ; 0 ; 3]$} & 14.8 & $2,1,2,3$ & $5,5,5,5$ & $4.10 \mathrm{E}-03$ \\
\hline 4412.8 & 40 & {$[6 ; 6 ; 1 ; 3]$} & 15.3 & $3,2,2,1$ & $4,4,4,4$ & $1.65 \mathrm{E}-03$ & 43 & {$[6 ; 6 ; 1 ; 3]$} & 14.8 & $3,1,3,3$ & $4,4,4,4$ & $4.60 \mathrm{E}-03$ \\
\hline 4719.9 & & & & & & & 44 & {$[6 ; 7 ; 0 ; 4]$} & 15.9 & $0,0,0,3$ & $0,0,0,4$ & $2.53 \mathrm{E}-03$ \\
\hline 4865.9 & 41 & {$[6 ; 7 ; 1 ; 4]$} & 16.9 & $0,0,1,0$ & $0,0,4,0$ & 8.04E-03 & 45 & {$[6 ; 7 ; 1 ; 4]$} & 14.8 & $0,0,0,1$ & $0,0,0,4$ & $2.47 \mathrm{E}-02$ \\
\hline \multicolumn{13}{|c|}{ KKKEDALN*ETR $(\alpha 64 N)$} \\
\hline 3535.5 & 42 & {$[4 ; 5 ; 0 ; 2]$} & 13.0 & $2,2,2,2$ & $4,4,4,4$ & $2.17 \mathrm{E}-03$ & 46 & {$[4 ; 5 ; 0 ; 2]$} & 12.7 & $3,0,0,0$ & $4,0,0,0$ & $6.06 \mathrm{E}-03$ \\
\hline 3900.6 & & & & & & & 47 & {$[5 ; 6 ; 0 ; 2]$} & 12.9 & $2,0,0,0$ & $4,0,0,0$ & $9.58 \mathrm{E}-04$ \\
\hline 4191.7 & 43 & {$[5 ; 6 ; 0 ; 3]$} & 14.5 & $1,2,0,0$ & $4,4,0,0$ & $1.88 \mathrm{E}-02$ & 48 & {$[5 ; 6 ; 0 ; 3]$} & 13.4 & $5,0,1,1$ & $4,0,4,4$ & $2.43 \mathrm{E}-03$ \\
\hline \multirow[t]{2}{*}{4337.8} & 44 & {$[5 ; 6 ; 1 ; 3]$} & 13.8 & $3,2,1,1$ & $4,4,4,4$ & $3.16 \mathrm{E}-03$ & 49 & {$[5 ; 6 ; 1 ; 3]$} & 13.5 & $3,1,1,0$ & $4,4,4,0$ & 7.08E-03 \\
\hline & & {$[5 ; 6 ; 1 ; 3]$} & 13.3 & $0,0,1,0$ & $0,0,5,0$ & $9.79 \mathrm{E}-03$ & & {$[5 ; 6 ; 1 ; 3]$} & 13.6 & $1,0,0,0$ & $5,0,0,0$ & $2.52 \mathrm{E}-03$ \\
\hline 4338.8 & & & & & & & 50 & {$[5 ; 6 ; 3 ; 2]$} & 13.4 & $1,0,0,1$ & $4,0,0,4$ & $2.30 \mathrm{E}-02$ \\
\hline 4208.7 & 45 & {$[5 ; 7 ; 1 ; 2]$} & 15.1 & $0,0,1,0$ & $0,0,3,0$ & $4.16 \mathrm{E}-02$ & & & & & & \\
\hline 4354.8 & & & & & & & 51 & {$[5 ; 7 ; 2 ; 2]$} & 15.1 & $1,0,0,0$ & $4,0,0,0$ & $4.54 \mathrm{E}-02$ \\
\hline \multirow[t]{2}{*}{4361.8} & & & & & & & 52 & {$[6 ; 4 ; 0 ; 4]$} & 13.6 & $1,0,0,0$ & $4,0,0,0$ & $3.33 \mathrm{E}-03$ \\
\hline & & & & & & & & {$[6 ; 4 ; 0 ; 4]$} & 14.3 & $1,0,0,0$ & $5,0,0,0$ & $6.90 \mathrm{E}-03$ \\
\hline 4362.8 & & & & & & & 53 & {$[6 ; 4 ; 2 ; 3]$} & 13.9 & $7,0,1,1$ & $4,0,4,4$ & $2.84 \mathrm{E}-02$ \\
\hline \multirow[t]{2}{*}{4394.8} & 46 & {$[6 ; 6 ; 0 ; 3]$} & 13.8 & $3,2,3,1$ & $4,4,4,4$ & $1.70 \mathrm{E}-03$ & 54 & {$[6 ; 6 ; 0 ; 3]$} & 13.3 & $5,2,1,2$ & $4,4,4,4$ & $3.66 \mathrm{E}-03$ \\
\hline & & {$[6 ; 6 ; 0 ; 3]$} & 13.6 & $1,0,1,0$ & $5,0,5,0$ & $1.06 \mathrm{E}-03$ & & {$[6 ; 6 ; 0 ; 3]$} & 13.0 & $1,0,0,1$ & $5,0,0,5$ & $1.69 \mathrm{E}-03$ \\
\hline \multicolumn{13}{|c|}{ ELPGVCN*ETMMALWEECKPCLK $(\alpha 81 N)$} \\
\hline 4916.0 & & & & & & & 55 & {$[4 ; 6 ; 1 ; 1]$} & 46.9 & $1,0,0,1$ & $4,0,0,4$ & $2.74 \mathrm{E}-02$ \\
\hline 4957.0 & & & & & & & 56 & {$[5 ; 5 ; 1 ; 1]$} & 50.7 & $0,0,0,1$ & $0,0,0,4$ & $1.61 \mathrm{E}-02$ \\
\hline 5701.3 & & & & & & & 57 & {$[5 ; 6 ; 1 ; 3]$} & 40.2 & $0,1,1,0$ & $0,5,5,0$ & $3.11 \mathrm{E}-02$ \\
\hline \multirow[t]{2}{*}{5725.3} & 47 & {$[6 ; 4 ; 0 ; 4]$} & 42.5 & $1,1,3,1$ & $5,5,5,5$ & $1.28 \mathrm{E}-02$ & 58 & {$[6 ; 4 ; 0 ; 4]$} & 42.0 & $3,3,2,3$ & $5,5,5,4$ & $5.49 \mathrm{E}-03$ \\
\hline & & & & & & & & {$[6 ; 4 ; 0 ; 4]$} & 41.1 & $0,0,0,4$ & $0,0,0,5$ & 4.36E-03 \\
\hline
\end{tabular}




\begin{tabular}{|c|c|c|c|c|c|c|c|c|c|c|c|c|}
\hline \multirow[t]{3}{*}{5726.3} & 48 & {$[6 ; 4 ; 2 ; 3]$} & 42.2 & $2,1,1,7$ & $4,4,4,5$ & $2.35 \mathrm{E}-02$ & 59 & {$[6 ; 4 ; 2 ; 3]$} & 41.8 & $2,2,8,1$ & $4,4,5,4$ & $2.72 \mathrm{E}-02$ \\
\hline & & {$[6 ; 4 ; 2 ; 3]$} & 42.2 & $7,8,6,1$ & $5,5,5,6$ & $2.42 \mathrm{E}-02$ & & {$[6 ; 4 ; 2 ; 3]$} & 41.7 & $8,7,0,8$ & $5,5,0,5$ & $2.26 \mathrm{E}-02$ \\
\hline & & {$[6 ; 4 ; 2 ; 3]$} & 42.3 & $1,0,0,0$ & $6,0,0,0$ & $1.64 \mathrm{E}-02$ & & & & & & \\
\hline \multirow[t]{2}{*}{5758.3} & 49 & {$[6 ; 6 ; 0 ; 3]$} & 50.4 & $0,0,0,3$ & $0,0,0,5$ & $1.07 \mathrm{E}-02$ & 60 & {$[6 ; 6 ; 0 ; 3]$} & 45.3 & $0,1,0,1$ & $0,4,0,5$ & $1.95 \mathrm{E}-02$ \\
\hline & & & & & & & & {$[6 ; 6 ; 0 ; 3]$} & 50.0 & $0,1,0,0$ & $0,5,0,0$ & $7.22 \mathrm{E}-03$ \\
\hline 5759.3 & 50 & {$[6 ; 6 ; 2 ; 2]$} & 50.0 & $1,0,0,0$ & $4,0,0,0$ & $2.24 \mathrm{E}-02$ & & & & & & \\
\hline \multicolumn{13}{|c|}{ QLEEFLN*QSSPFYFWMNGDR ( $\alpha 123 N)$} \\
\hline 4736.9 & 51 & {$[3 ; 10 ; 0 ; 0]$} & 51.9 & $4,4,3,1$ & $4,4,4,4$ & $1.34 \mathrm{E}-02$ & 61 & {$[3 ; 10 ; 0 ; 0]$} & 51.7 & $0,1,1,0$ & $0,4,4,0$ & $2.79 \mathrm{E}-02$ \\
\hline 4420.8 & 52 & {$[4 ; 5 ; 0 ; 1]$} & 43.9 & $0,0,1,2$ & $0,0,4,4$ & $2.83 \mathrm{E}-02$ & 62 & {$[4 ; 5 ; 0 ; 1]$} & 44.0 & $0,0,1,0$ & $0,0,4,0$ & $2.43 \mathrm{E}-02$ \\
\hline \multirow[t]{3}{*}{4711.9} & 53 & {$[4 ; 5 ; 0 ; 2]$} & 47.4 & $1,1,3,5$ & $3,3,4,4$ & $1.95 \mathrm{E}-02$ & 63 & {$[4 ; 5 ; 0 ; 2]$} & 47.0 & $2,3,3,2$ & $4,4,4,4$ & 2.07E-02 \\
\hline & & {$[4 ; 5 ; 0 ; 2]$} & 47.3 & $3,5,0,4$ & $4,4,0,5$ & $2.46 \mathrm{E}-02$ & & {$[4 ; 5 ; 0 ; 2]$} & 47.0 & $0,0,2,0$ & $0,0,5,0$ & $2.42 \mathrm{E}-02$ \\
\hline & & {$[4 ; 5 ; 0 ; 2]$} & 47.3 & $3,1,0,0$ & $5,5,0,0$ & $2.60 \mathrm{E}-02$ & & & & & & \\
\hline 4566.9 & 54 & {$[4 ; 5 ; 1 ; 1]$} & 47.9 & $0,0,1,0$ & $0,0,4,0$ & $3.71 \mathrm{E}-02$ & 64 & {$[4 ; 5 ; 1 ; 1]$} & 46.5 & $0,0,0,1$ & $0,0,0,4$ & 4.34E-02 \\
\hline 4858.0 & 55 & {$[4 ; 5 ; 1 ; 2]$} & 47.3 & $3,4,3,1$ & $4,4,4,4$ & $2.26 \mathrm{E}-02$ & 65 & {$[4 ; 5 ; 1 ; 2]$} & 46.8 & $0,0,1,0$ & $0,0,4,0$ & $4.48 \mathrm{E}-02$ \\
\hline \multirow[t]{2}{*}{4712.9} & 56 & {$[4 ; 5 ; 2 ; 1]$} & 46.9 & $1,0,1,0$ & $3,0,4,0$ & $4.68 \mathrm{E}-02$ & 66 & {$[4 ; 5 ; 2 ; 1]$} & 47.2 & $0,1,0,0$ & $0,3,0,0$ & $3.99 \mathrm{E}-02$ \\
\hline & & {$[4 ; 5 ; 2 ; 1]$} & 46.6 & $2,0,0,0$ & $4,0,0,0$ & $3.66 \mathrm{E}-02$ & & {$[4 ; 5 ; 2 ; 1]$} & 47.0 & $0,1,0,0$ & $0,4,0,0$ & $4.20 \mathrm{E}-02$ \\
\hline 4859.0 & 57 & {$[4 ; 5 ; 3 ; 1]$} & 45.1 & $0,1,2,1$ & $0,4,4,4$ & $3.04 \mathrm{E}-02$ & 67 & {$[4 ; 5 ; 3 ; 1]$} & 47.2 & $0,0,1,0$ & $0,0,4,0$ & 4.39E-02 \\
\hline 4728.9 & & & & & & & 68 & {$[4 ; 6 ; 1 ; 1]$} & 47.4 & $1,0,0,0$ & $4,0,0,0$ & $3.66 \mathrm{E}-02$ \\
\hline \multirow[t]{2}{*}{4736.9} & 58 & {$[5 ; 3 ; 1 ; 2]$} & 51.8 & $3,3,0,0$ & $4,4,0,0$ & $3.47 \mathrm{E}-02$ & & & & & & \\
\hline & 59 & {$[5 ; 5 ; 0 ; 1]$} & 47.0 & $0,1,0,0$ & $0,4,0,0$ & 4.54E-02 & 69 & {$[5 ; 5 ; 0 ; 1]$} & 47.0 & $0,0,1,1$ & $0,0,5,4$ & $4.02 \mathrm{E}-02$ \\
\hline 4915.0 & 60 & {$[5 ; 5 ; 0 ; 2]$} & 47.5 & $3,2,0,2$ & $4,4,0,4$ & $2.10 \mathrm{E}-02$ & 70 & {$[5 ; 5 ; 0 ; 2]$} & 47.4 & $2,1,2,0$ & $4,4,4,0$ & $1.65 \mathrm{E}-02$ \\
\hline 4769.9 & 62 & {$[5 ; 5 ; 1 ; 1]$} & 48.3 & $0,1,0,1$ & $0,4,0,4$ & $3.75 \mathrm{E}-02$ & 71 & {$[5 ; 5 ; 1 ; 1]$} & 52.8 & $0,2,2,8$ & $0,4,4,4$ & $3.38 \mathrm{E}-02$ \\
\hline 5368.1 & 62 & {$[5 ; 6 ; 0 ; 3]$} & 51.3 & $0,1,0,0$ & $0,4,0,0$ & $3.51 \mathrm{E}-03$ & & & & & & \\
\hline 6024.3 & & & & & & & 72 & {$[6 ; 7 ; 0 ; 4]$} & 45.9 & $0,0,0,1$ & $0,0,0,5$ & $5.04 \mathrm{E}-02$ \\
\hline \multicolumn{13}{|c|}{ HN*STGCLR (B269N) } \\
\hline 3173.2 & & & & & & & 73 & {$[3 ; 10 ; 0 ; 0]$} & 13.4 & $0,1,0,0$ & $0,3,0,0$ & $3.00 \mathrm{E}-02$ \\
\hline 3133.2 & 63 & {$[4 ; 4 ; 3 ; 1]$} & 12.9 & $0,0,0,1$ & $0,0,0,3$ & $2.80 \mathrm{E}-02$ & 74 & {$[4 ; 4 ; 3 ; 1]$} & 12.2 & $3,0,0,0$ & $3,0,0,0$ & $2.29 \mathrm{E}-02$ \\
\hline \multirow[t]{2}{*}{2857.1} & 64 & {$[4 ; 5 ; 0 ; 1]$} & 12.3 & $107,85,78,124$ & $3,3,3,3$ & $3.13 \mathrm{E}-04$ & 75 & {$[4 ; 5 ; 0 ; 1]$} & 11.8 & $52,82,42,16$ & $3,3,3,3$ & $2.90 \mathrm{E}-04$ \\
\hline & & {$[4 ; 5 ; 0 ; 1]$} & 11.6 & $3,8,2,44$ & $4,4,4,4$ & $1.23 \mathrm{E}-03$ & & {$[4 ; 5 ; 0 ; 1]$} & 11.5 & $1,2,1,3$ & $4,4,4,4$ & $1.56 \mathrm{E}-03$ \\
\hline
\end{tabular}




\begin{tabular}{|c|c|c|c|c|c|c|c|c|c|c|c|c|}
\hline \multirow[t]{2}{*}{3148.2} & 65 & {$[4 ; 5 ; 0 ; 2]$} & 13.1 & $10,12,15,12$ & $3,3,3,3$ & $7.11 \mathrm{E}-04$ & 76 & {$[4 ; 5 ; 0 ; 2]$} & 12.6 & $16,14,11,8$ & $3,3,3,3$ & $1.05 \mathrm{E}-03$ \\
\hline & & {$[4 ; 5 ; 0 ; 2]$} & 13.1 & $6,9,12,12$ & $4,4,4,4$ & $1.30 \mathrm{E}-03$ & & {$[4 ; 5 ; 0 ; 2]$} & 12.6 & $8,9,8,7$ & $4,4,4,4$ & $1.50 \mathrm{E}-03$ \\
\hline 3003.2 & 66 & {$[4 ; 5 ; 1 ; 1]$} & 12.6 & $2,2,3,2$ & $3,3,3,3$ & $5.44 \mathrm{E}-03$ & 77 & {$[4 ; 5 ; 1 ; 1]$} & 12.2 & $6,1,0,3$ & $3,3,0,3$ & $8.29 \mathrm{E}-03$ \\
\hline \multirow[t]{2}{*}{3294.3} & 67 & {$[4 ; 5 ; 1 ; 2]$} & 12.7 & $2,2,1,1$ & $3,3,3,3$ & $1.41 \mathrm{E}-02$ & 78 & {$[4 ; 5 ; 1 ; 2]$} & 13.0 & $4,1,0,1$ & $3,3,0,3$ & $9.28 \mathrm{E}-03$ \\
\hline & & & & & & & & {$[4 ; 5 ; 1 ; 2]$} & 13.2 & $5,0,0,2$ & $4,0,0,4$ & $1.58 \mathrm{E}-02$ \\
\hline \multirow[t]{2}{*}{3295.3} & 69 & {$[4 ; 5 ; 3 ; 1]$} & 12.2 & $6,2,1,0$ & $3,3,3,0$ & $2.43 \mathrm{E}-02$ & 79 & {$[4 ; 5 ; 3 ; 1]$} & 11.5 & $11,0,0,1$ & $3,0,0,3$ & $2.79 \mathrm{E}-02$ \\
\hline & & & & & & & & {$[4 ; 5 ; 3 ; 1]$} & 11.9 & $3,0,0,0$ & $4,0,0,0$ & $2.83 \mathrm{E}-02$ \\
\hline 3165.2 & & & & & & & 80 & {$[4 ; 6 ; 1 ; 1]$} & 12.5 & $0,1,0,0$ & $0,3,0,0$ & $1.51 \mathrm{E}-02$ \\
\hline 3173.2 & & & & & & & 81 & {$[5 ; 3 ; 1 ; 2]$} & 13.4 & $0,1,0,0$ & $0,3,0,0$ & 7.69E-03 \\
\hline \multirow[t]{2}{*}{3060.2} & 70 & {$[5 ; 5 ; 0 ; 1]$} & 12.7 & $3,2,2,2$ & $3,3,3,3$ & $7.17 \mathrm{E}-03$ & 82 & {$[5 ; 5 ; 0 ; 1]$} & 12.2 & $5,2,2,1$ & $3,3,3,3$ & $1.27 \mathrm{E}-02$ \\
\hline & & {$[5 ; 5 ; 0 ; 1]$} & 12.9 & $0,0,0,1$ & $0,0,0,4$ & $1.12 \mathrm{E}-02$ & & & & & & \\
\hline \multirow[t]{2}{*}{3351.3} & 71 & {$[5 ; 5 ; 0 ; 2]$} & 13.2 & $2,2,0,2$ & $3,3,0,4$ & $1.26 \mathrm{E}-02$ & 83 & {$[5 ; 5 ; 0 ; 2]$} & 12.4 & $8,0,2,1$ & $3,0,4,3$ & $1.51 \mathrm{E}-02$ \\
\hline & & {$[5 ; 5 ; 0 ; 2]$} & 12.0 & $2,0,0,0$ & $4,0,0,0$ & $8.80 \mathrm{E}-03$ & & {$[5 ; 5 ; 0 ; 2]$} & 13.6 & $2,0,0,0$ & $4,0,0,0$ & $1.58 \mathrm{E}-02$ \\
\hline 3206.2 & & & & & & & 84 & {$[5 ; 5 ; 1 ; 1]$} & 12.8 & $1,0,0,1$ & $3,0,0,3$ & $2.25 \mathrm{E}-02$ \\
\hline \multirow[t]{2}{*}{3513.3} & 72 & {$[5 ; 6 ; 0 ; 2]$} & 12.6 & $9,5,7,4$ & $3,3,3,3$ & $1.34 \mathrm{E}-03$ & 85 & {$[5 ; 6 ; 0 ; 2]$} & 12.1 & $12,5,4,6$ & $3,3,3,3$ & $5.31 \mathrm{E}-04$ \\
\hline & & {$[5 ; 6 ; 0 ; 2]$} & 12.7 & $6,8,5,9$ & $4,4,4,4$ & $8.71 \mathrm{E}-04$ & & {$[5 ; 6 ; 0 ; 2]$} & 12.1 & $10,3,9,6$ & $4,4,4,4$ & $1.18 \mathrm{E}-03$ \\
\hline \multirow[t]{2}{*}{3804.4} & 73 & {$[5 ; 6 ; 0 ; 3]$} & 14.5 & $2,1,1,1$ & $3,4,4,4$ & $3.27 \mathrm{E}-03$ & 86 & {$[5 ; 6 ; 0 ; 3]$} & 13.9 & $5,1,0,3$ & $3,3,0,4$ & $2.44 \mathrm{E}-03$ \\
\hline & & {$[5 ; 6 ; 0 ; 3]$} & 14.5 & $2,0,0,0$ & $4,0,0,0$ & 8.33E-03 & & {$[5 ; 6 ; 0 ; 3]$} & 14.3 & $6,2,0,0$ & $4,4,0,0$ & $1.99 \mathrm{E}-03$ \\
\hline \multirow[t]{2}{*}{3659.4} & 74 & {$[5 ; 6 ; 1 ; 2]$} & 12.4 & $1,1,0,1$ & $3,4,0,4$ & $6.47 \mathrm{E}-03$ & 87 & {$[5 ; 6 ; 1 ; 2]$} & 11.9 & $3,0,0,1$ & $3,0,0,4$ & $9.59 \mathrm{E}-03$ \\
\hline & & {$[5 ; 6 ; 1 ; 2]$} & 12.3 & $2,0,0,0$ & $4,0,0,0$ & $8.81 \mathrm{E}-05$ & & & & & & \\
\hline 3951.5 & & & & & & & 88 & {$[5 ; 6 ; 3 ; 2]$} & 13.6 & $2,0,0,0$ & $4,0,0,0$ & $1.96 \mathrm{E}-02$ \\
\hline \multirow[t]{2}{*}{3975.5} & 75 & {$[6 ; 4 ; 2 ; 3]$} & 14.9 & $0,0,2,0$ & $0,0,4,0$ & $2.67 \mathrm{E}-02$ & 89 & {$[6 ; 4 ; 2 ; 3]$} & 14.6 & $1,0,3,3$ & $3,0,4,3$ & $2.47 \mathrm{E}-02$ \\
\hline & & & & & & & & {$[6 ; 4 ; 2 ; 3]$} & 14.5 & $2,0,0,6$ & $4,0,0,4$ & $2.95 \mathrm{E}-02$ \\
\hline \multirow[t]{2}{*}{3716.4} & 76 & {$[6 ; 6 ; 0 ; 2]$} & 12.7 & $1,0,0,0$ & $3,0,0,0$ & $1.28 \mathrm{E}-02$ & 90 & {$[6 ; 6 ; 0 ; 2]$} & 13.1 & $2,0,0,0$ & $3,0,0,0$ & $4.65 \mathrm{E}-03$ \\
\hline & & & & & & & & {$[6 ; 6 ; 0 ; 2]$} & 13.1 & $2,0,0,0$ & $4,0,0,0$ & $4.82 \mathrm{E}-03$ \\
\hline 3717.4 & & & & & & & 91 & {$[6 ; 6 ; 2 ; 1]$} & 12.6 & $2,0,0,0$ & $4,0,0,0$ & $1.04 \mathrm{E}-02$ \\
\hline 4008.5 & & & & & & & 92 & {$[6 ; 6 ; 2 ; 2]$} & 13.6 & $2,0,0,0$ & $4,0,0,0$ & $1.01 \mathrm{E}-03$ \\
\hline \multicolumn{13}{|c|}{ MLN*TSSLLEQLNEQFNWVSR ( $(3332 N)$} \\
\hline 4598.0 & & & & & & & 93 & {$[4 ; 4 ; 3 ; 1]$} & 64.7 & $0,1,0,1$ & $0,4,0,4$ & $2.41 \mathrm{E}-02$ \\
\hline
\end{tabular}




\begin{tabular}{|c|c|c|c|c|c|c|c|c|c|c|c|c|}
\hline 4614.0 & 77 & {$[4 ; 5 ; 2 ; 1]$} & 73.4 & $0,1,0,0$ & $0,4,0,0$ & $3.80 \mathrm{E}-02$ & 94 & {$[4 ; 5 ; 2 ; 1]$} & 65.2 & $0,1,0,1$ & $0,4,0,4$ & $1.82 \mathrm{E}-02$ \\
\hline 4524.9 & 78 & {$[5 ; 5 ; 0 ; 1]$} & 69.7 & $2,2,1,2$ & $4,4,4,4$ & $1.97 \mathrm{E}-03$ & 95 & {$[5 ; 5 ; 0 ; 1]$} & 69.7 & $2,2,1,2$ & $4,4,4,4$ & $6.12 \mathrm{E}-03$ \\
\hline 4816.0 & 79 & {$[5 ; 5 ; 0 ; 2]$} & 72.4 & $1,3,0,0$ & $4,4,0,0$ & $2.81 \mathrm{E}-03$ & 96 & {$[5 ; 5 ; 0 ; 2]$} & 72.4 & $3,2,3,1$ & $4,4,4,4$ & 4.14E-03 \\
\hline 4671.0 & 80 & {$[5 ; 5 ; 1 ; 1]$} & 66.0 & $1,2,2,2$ & $4,4,4,4$ & $2.87 \mathrm{E}-02$ & 97 & {$[5 ; 5 ; 1 ; 1]$} & 64.6 & $2,3,1,4$ & $4,4,4,4$ & $3.11 \mathrm{E}-02$ \\
\hline 5181.2 & 81 & {$[6 ; 6 ; 0 ; 2]$} & 72.8 & $1,0,0,0$ & $4,0,0,0$ & $1.01 \mathrm{E}-03$ & & & & & & \\
\hline \multicolumn{13}{|c|}{ LAN*LTQGEDQYYLR ( $\beta 352 N)$} \\
\hline \multirow[t]{2}{*}{3912.6} & 82 & {$[3 ; 10 ; 0 ; 0]$} & 49.3 & $0,1,1,0$ & $0,4,3,0$ & $1.71 \mathrm{E}-02$ & 98 & {$[3 ; 10 ; 0 ; 0]$} & 49.0 & $0,1,1,1$ & $0,4,4,3$ & $2.12 \mathrm{E}-02$ \\
\hline & & & & & & & & {$[3 ; 10 ; 0 ; 0]$} & 49.9 & $0,0,0,1$ & $0,0,0,4$ & $1.65 \mathrm{E}-02$ \\
\hline \multirow[t]{3}{*}{3887.6} & 83 & {$[4 ; 5 ; 0 ; 2]$} & 44.4 & $11,10,11,10$ & $3,3,3,3$ & $2.80 \mathrm{E}-03$ & 99 & {$[4 ; 5 ; 0 ; 2]$} & 44.2 & $7,8,5,5$ & $3,3,3,3$ & $2.14 \mathrm{E}-03$ \\
\hline & & {$[4 ; 5 ; 0 ; 2]$} & 44.4 & $14,14,12,19$ & $4,4,4,4$ & $2.05 \mathrm{E}-03$ & & {$[4 ; 5 ; 0 ; 2]$} & 44.2 & $9,10,10,6$ & $4,4,4,4$ & $2.07 \mathrm{E}-03$ \\
\hline & & {$[4 ; 5 ; 0 ; 2]$} & 44.4 & $3,3,2,4$ & $5,5,5,5$ & $1.13 \mathrm{E}-03$ & & {$[4 ; 5 ; 0 ; 2]$} & 44.3 & $2,1,3,0$ & $5,5,5,0$ & $3.87 \mathrm{E}-03$ \\
\hline \multirow[t]{3}{*}{4033.7} & 84 & {$[4 ; 5 ; 1 ; 2]$} & 44.4 & $4,3,3,6$ & $3,3,3,3$ & $3.12 \mathrm{E}-03$ & 100 & {$[4 ; 5 ; 1 ; 2]$} & 44.2 & $2,3,2,0$ & $3,3,3,0$ & 4.70E-03 \\
\hline & & {$[4 ; 5 ; 1 ; 2]$} & 44.4 & $3,4,3,12$ & $4,4,4,4$ & 2.99E-03 & & {$[4 ; 5 ; 1 ; 2]$} & 44.2 & $2,3,3,0$ & $4,4,4,0$ & 4.08E-03 \\
\hline & & {$[4 ; 5 ; 1 ; 2]$} & 44.0 & $0,0,0,1$ & $0,0,0,5$ & 1.19E-03 & & & & & & \\
\hline 3888.6 & 85 & {$[4 ; 5 ; 2 ; 1]$} & 46.0 & $1,0,0,0$ & $4,0,0,0$ & $1.58 \mathrm{E}-02$ & & & & & & \\
\hline \multirow[t]{2}{*}{3904.6} & 86 & {$[4 ; 6 ; 1 ; 1]$} & 44.4 & $5,3,3,4$ & $3,3,3,3$ & $1.22 \mathrm{E}-02$ & 101 & {$[4 ; 6 ; 1 ; 1]$} & 44.3 & $3,2,1,0$ & $3,3,3,0$ & $1.31 \mathrm{E}-02$ \\
\hline & & {$[4 ; 6 ; 1 ; 1]$} & 44.4 & $4,3,3,3$ & $4,4,4,4$ & 1.29E-02 & & {$[4 ; 6 ; 1 ; 1]$} & 44.3 & $3,4,2,0$ & $4,4,4,0$ & $1.13 \mathrm{E}-02$ \\
\hline \multirow[t]{2}{*}{3912.6} & 87 & {$[5 ; 3 ; 1 ; 2]$} & 49.3 & $0,1,1,0$ & $0,4,3,0$ & 2.07E-02 & 102 & {$[5 ; 3 ; 1 ; 2]$} & 49.0 & $0,1,1,1$ & $0,4,4,3$ & $1.65 \mathrm{E}-02$ \\
\hline & & & & & & & & {$[5 ; 3 ; 1 ; 2]$} & 49.9 & $0,0,0,1$ & $0,0,0,4$ & $2.12 \mathrm{E}-02$ \\
\hline \multirow[t]{2}{*}{4090.7} & 88 & {$[5 ; 5 ; 0 ; 2]$} & 44.6 & $4,4,5,4$ & $3,3,3,3$ & 4.64E-03 & 103 & {$[5 ; 5 ; 0 ; 2]$} & 44.4 & $3,5,5,2$ & $3,3,3,4$ & 4.61E-03 \\
\hline & & {$[5 ; 5 ; 0 ; 2]$} & 44.6 & $6,6,6,8$ & $4,4,4,4$ & $2.62 \mathrm{E}-03$ & & {$[5 ; 5 ; 0 ; 2]$} & 44.5 & $5,4,6,0$ & $4,4,4,0$ & $2.69 \mathrm{E}-03$ \\
\hline 3945.6 & 89 & {$[5 ; 5 ; 1 ; 1]$} & 47.9 & $0,1,0,1$ & $0,3,0,4$ & $2.53 \mathrm{E}-02$ & & & & & & \\
\hline \multirow[t]{2}{*}{4252.7} & 90 & {$[5 ; 6 ; 0 ; 2]$} & 44.2 & $2,2,2,1$ & $4,4,3,3$ & $1.06 \mathrm{E}-03$ & 104 & {$[5 ; 6 ; 0 ; 2]$} & 44.0 & $1,1,1,0$ & $3,3,3,0$ & $2.02 \mathrm{E}-03$ \\
\hline & & {$[5 ; 6 ; 0 ; 2]$} & 44.2 & $0,0,2,5$ & $0,0,4,4$ & $5.12 \mathrm{E}-03$ & & {$[5 ; 6 ; 0 ; 2]$} & 44.0 & $2,3,3,0$ & $4,4,4,0$ & $2.54 \mathrm{E}-03$ \\
\hline \multirow[t]{4}{*}{4543.8} & 91 & {$[5 ; 6 ; 0 ; 3]$} & 47.5 & $5,6,5,5$ & $3,3,3,3$ & $2.44 \mathrm{E}-03$ & 105 & {$[5 ; 6 ; 0 ; 3]$} & 47.2 & $4,4,3,5$ & $3,3,3,3$ & $1.92 \mathrm{E}-03$ \\
\hline & & {$[5 ; 6 ; 0 ; 3]$} & 47.5 & $22,28,33,20$ & $4,4,4,4$ & $2.65 \mathrm{E}-03$ & & {$[5 ; 6 ; 0 ; 3]$} & 47.2 & $17,14,20,15$ & $4,4,4,4$ & 4.50E-03 \\
\hline & & {$[5 ; 6 ; 0 ; 3]$} & 47.4 & $3,4,4,5$ & $5,5,5,5$ & $1.95 \mathrm{E}-03$ & & {$[5 ; 6 ; 0 ; 3]$} & 47.1 & $1,2,3,3$ & $5,5,5,5$ & $2.42 \mathrm{E}-03$ \\
\hline & & {$[5 ; 6 ; 0 ; 3]$} & 47.7 & $0,0,0,1$ & $0,0,0,6$ & 4.76E-03 & & & & & & \\
\hline 4398.8 & 92 & {$[5 ; 6 ; 1 ; 2]$} & 44.1 & $1,1,1,1$ & $4,4,4,3$ & $1.96 \mathrm{E}-03$ & 106 & {$[5 ; 6 ; 1 ; 2]$} & 44.0 & $0,1,1,0$ & $0,4,4,0$ & $4.21 \mathrm{E}-03$ \\
\hline
\end{tabular}




\begin{tabular}{|c|c|c|c|c|c|c|c|c|c|c|c|c|}
\hline & & {$[5 ; 6 ; 1 ; 2]$} & 44.2 & $0,0,0,4$ & $0,0,0,4$ & $3.03 \mathrm{E}-03$ & & & & & & \\
\hline \multirow[t]{3}{*}{4689.9} & 93 & {$[5 ; 6 ; 1 ; 3]$} & 47.3 & $4,6,4,4$ & $3,3,3,3$ & $5.64 \mathrm{E}-03$ & 107 & {$[5 ; 6 ; 1 ; 3]$} & 47.0 & $3,3,3,4$ & $3,3,3,3$ & $5.45 \mathrm{E}-03$ \\
\hline & & {$[5 ; 6 ; 1 ; 3]$} & 47.4 & $19,29,31,20$ & $4,4,4,4$ & $3.14 \mathrm{E}-03$ & & {$[5 ; 6 ; 1 ; 3]$} & 47.0 & $9,9,8,12$ & $4,4,4,4$ & $4.60 \mathrm{E}-03$ \\
\hline & & {$[5 ; 6 ; 1 ; 3]$} & 47.3 & $4,3,4,8$ & $5,5,5,5$ & $1.82 \mathrm{E}-03$ & & {$[5 ; 6 ; 1 ; 3]$} & 46.9 & $2,1,4,3$ & $5,5,5,5$ & $2.28 \mathrm{E}-03$ \\
\hline 4544.8 & 94 & {$[5 ; 6 ; 2 ; 2]$} & 44.0 & $0,0,0,1$ & $0,0,0,4$ & $3.57 \mathrm{E}-03$ & 108 & {$[5 ; 6 ; 2 ; 2]$} & 47.6 & $0,1,0,0$ & $0,5,0,0$ & $1.26 \mathrm{E}-02$ \\
\hline \multirow[t]{3}{*}{4835.9} & 95 & {$[5 ; 6 ; 2 ; 3]$} & 47.2 & $2,1,1,8$ & $3,3,3,4$ & 2.64E-03 & 109 & {$[5 ; 6 ; 2 ; 3]$} & 46.9 & $2,5,5,6$ & $4,4,4,4$ & $5.93 \mathrm{E}-03$ \\
\hline & & {$[5 ; 6 ; 2 ; 3]$} & 47.3 & $9,9,7,4$ & $4,4,4,5$ & 4.42E-03 & & {$[5 ; 6 ; 2 ; 3]$} & 47.0 & $0,0,2,0$ & $0,0,5,0$ & $1.54 \mathrm{E}-03$ \\
\hline & & {$[5 ; 6 ; 2 ; 3]$} & 47.2 & $1,0,0,0$ & $5,0,0,0$ & $1.16 \mathrm{E}-03$ & & & & & & \\
\hline 4690.9 & 96 & {$[5 ; 6 ; 3 ; 2]$} & 48.2 & $0,0,1,0$ & $0,0,4,0$ & $1.68 \mathrm{E}-02$ & 110 & {$[5 ; 6 ; 3 ; 2]$} & 46.3 & $0,0,0,1$ & $0,0,0,5$ & $1.33 \mathrm{E}-02$ \\
\hline \multirow[t]{2}{*}{4560.8} & 97 & {$[5 ; 7 ; 1 ; 2]$} & 47.6 & $2,3,3,2$ & $3,3,3,4$ & $1.49 \mathrm{E}-02$ & 111 & {$[5 ; 7 ; 1 ; 2]$} & 47.1 & $2,1,2,2$ & $3,3,4,4$ & $1.00 \mathrm{E}-02$ \\
\hline & & {$[5 ; 7 ; 1 ; 2]$} & 47.4 & $3,2,1,0$ & $4,4,4,0$ & $1.40 \mathrm{E}-02$ & & {$[5 ; 7 ; 1 ; 2]$} & 47.3 & $4,3,0,0$ & $4,4,0,0$ & $1.33 \mathrm{E}-02$ \\
\hline \multirow[t]{2}{*}{4706.9} & 98 & {$[5 ; 7 ; 2 ; 2]$} & 47.3 & $2,2,4,2$ & $3,3,3,4$ & $1.35 \mathrm{E}-02$ & 112 & {$[5 ; 7 ; 2 ; 2]$} & 47.0 & $1,1,4,3$ & $4,3,4,4$ & $1.34 \mathrm{E}-02$ \\
\hline & & {$[5 ; 7 ; 2 ; 2]$} & 47.3 & $2,6,6,1$ & $4,4,4,5$ & $1.42 \mathrm{E}-02$ & & {$[5 ; 7 ; 2 ; 2]$} & 47.0 & $0,3,0,0$ & $0,4,0,0$ & $1.68 \mathrm{E}-02$ \\
\hline 4561.9 & & & & & & & 113 & {$[5 ; 7 ; 3 ; 1]$} & 47.2 & $1,0,1,0$ & $3,0,3,0$ & $1.04 \mathrm{E}-02$ \\
\hline 4714.9 & & & & & & & 114 & {$[6 ; 4 ; 2 ; 3]$} & 45.7 & $0,0,0,1$ & $0,0,0,4$ & $4.88 \mathrm{E}-03$ \\
\hline \multirow[t]{2}{*}{4455.8} & 99 & {$[6 ; 6 ; 0 ; 2]$} & 44.2 & $1,2,1,1$ & $3,4,3,3$ & $5.06 \mathrm{E}-03$ & 115 & {$[6 ; 6 ; 0 ; 2]$} & 44.1 & $3,3,4,0$ & $4,4,4,0$ & $5.36 \mathrm{E}-03$ \\
\hline & & {$[6 ; 6 ; 0 ; 2]$} & 44.2 & $2,0,2,5$ & $4,0,4,4$ & $4.58 \mathrm{E}-03$ & & & & & & \\
\hline \multirow[t]{3}{*}{4746.9} & 100 & {$[6 ; 6 ; 0 ; 3]$} & 47.5 & $6,6,6,5$ & $3,3,3,3$ & $3.22 \mathrm{E}-03$ & 116 & {$[6 ; 6 ; 0 ; 3]$} & 47.2 & $4,5,3,4$ & $3,3,3,3$ & $3.39 \mathrm{E}-03$ \\
\hline & & {$[6 ; 6 ; 0 ; 3]$} & 47.5 & $21,26,31,20$ & $4,4,4,4$ & $3.40 \mathrm{E}-03$ & & {$[6 ; 6 ; 0 ; 3]$} & 47.2 & $23,28,27,25$ & $4,4,4,4$ & $2.34 \mathrm{E}-03$ \\
\hline & & {$[6 ; 6 ; 0 ; 3]$} & 47.5 & $5,5,4,8$ & $5,5,5,5$ & $3.59 \mathrm{E}-03$ & & {$[6 ; 6 ; 0 ; 3]$} & 47.2 & $3,4,4,4$ & $5,5,5,5$ & $3.52 \mathrm{E}-03$ \\
\hline \multirow[t]{2}{*}{4893.0} & 101 & {$[6 ; 6 ; 1 ; 3]$} & 47.5 & $9,1,1,7$ & $4,3,3,4$ & 7.94E-03 & 117 & {$[6 ; 6 ; 1 ; 3]$} & 47.2 & $6,6,6,7$ & $4,4,4,4$ & $7.54 \mathrm{E}-03$ \\
\hline & & {$[6 ; 6 ; 1 ; 3]$} & 47.5 & $0,9,8,3$ & $0,4,4,5$ & $3.58 \mathrm{E}-03$ & & {$[6 ; 6 ; 1 ; 3]$} & 47.3 & $0,0,1,0$ & $0,0,5,0$ & $8.23 \mathrm{E}-03$ \\
\hline 4747.9 & 102 & {$[6 ; 6 ; 2 ; 2]$} & 49.6 & $0,0,1,0$ & $0,0,4,0$ & $1.64 \mathrm{E}-02$ & 118 & {$[6 ; 6 ; 2 ; 2]$} & 46.5 & $0,0,0,1$ & $0,0,0,5$ & $1.16 \mathrm{E}-02$ \\
\hline
\end{tabular}

Table S1. Glycopeptides assignments after MS1 deconvolution. Key [HexNAc; Hex; Fuc; Neu5Ac]. 

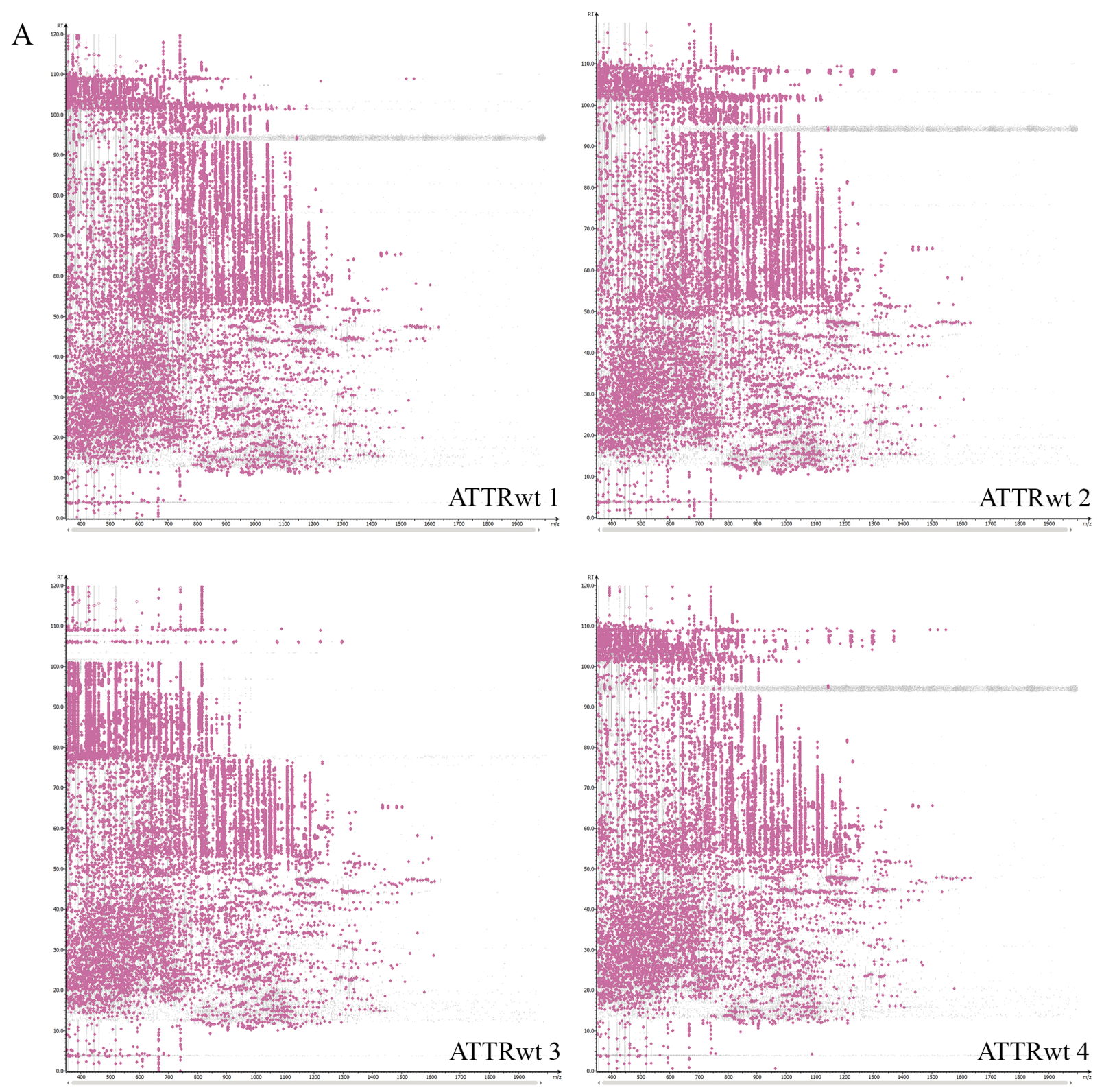

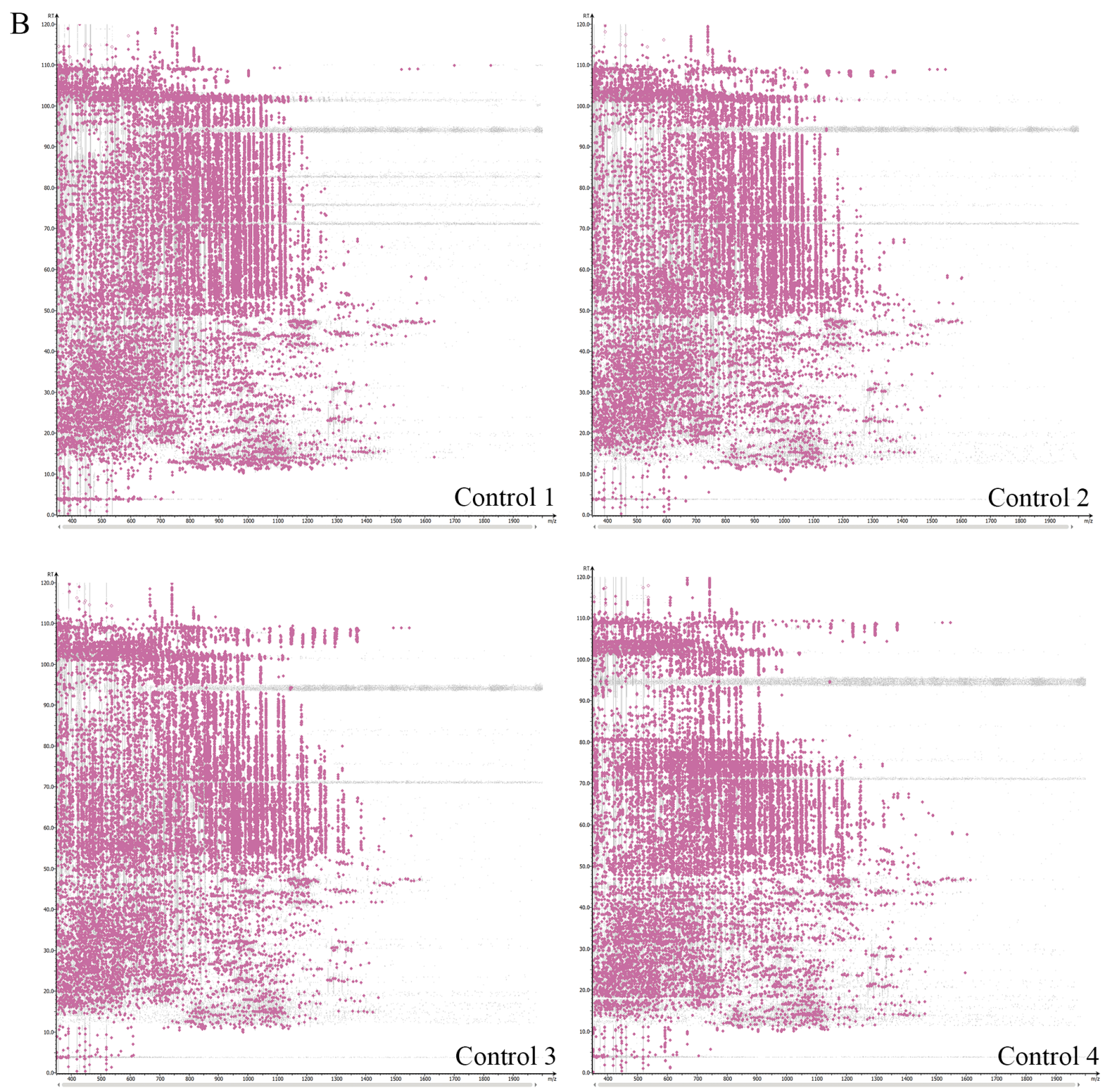


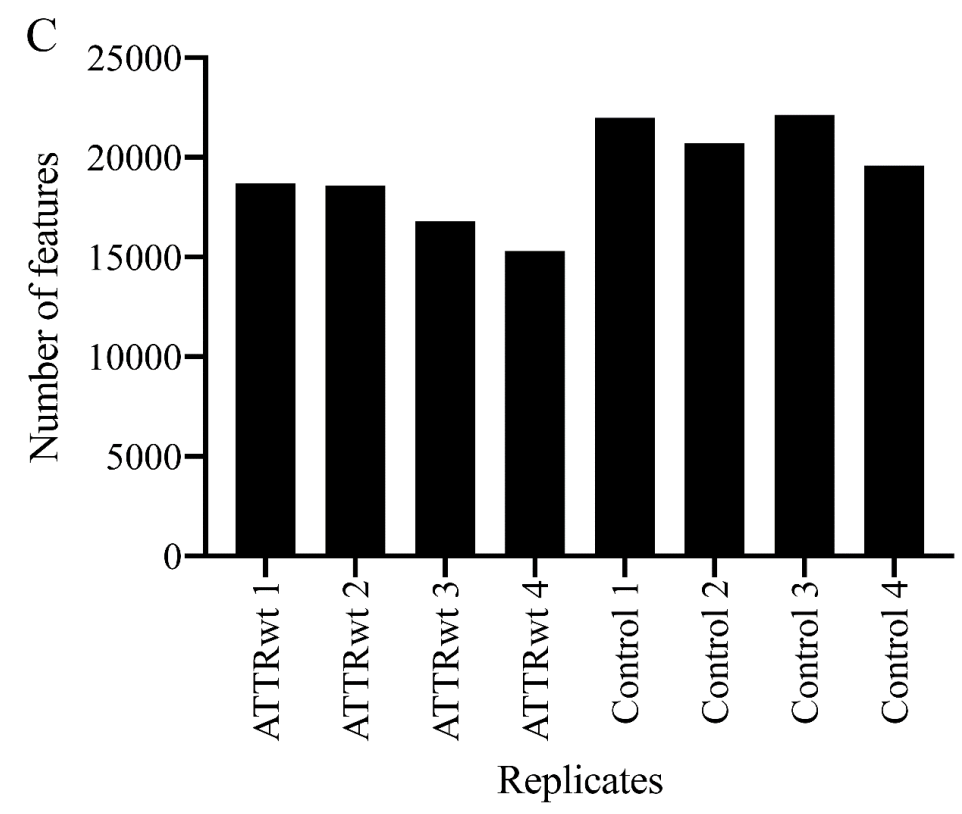

Figure S1. LC-MS features in ATTRwt and control CLU. Map plots (A, B) and number of features per replicate $(\mathrm{C})$. 


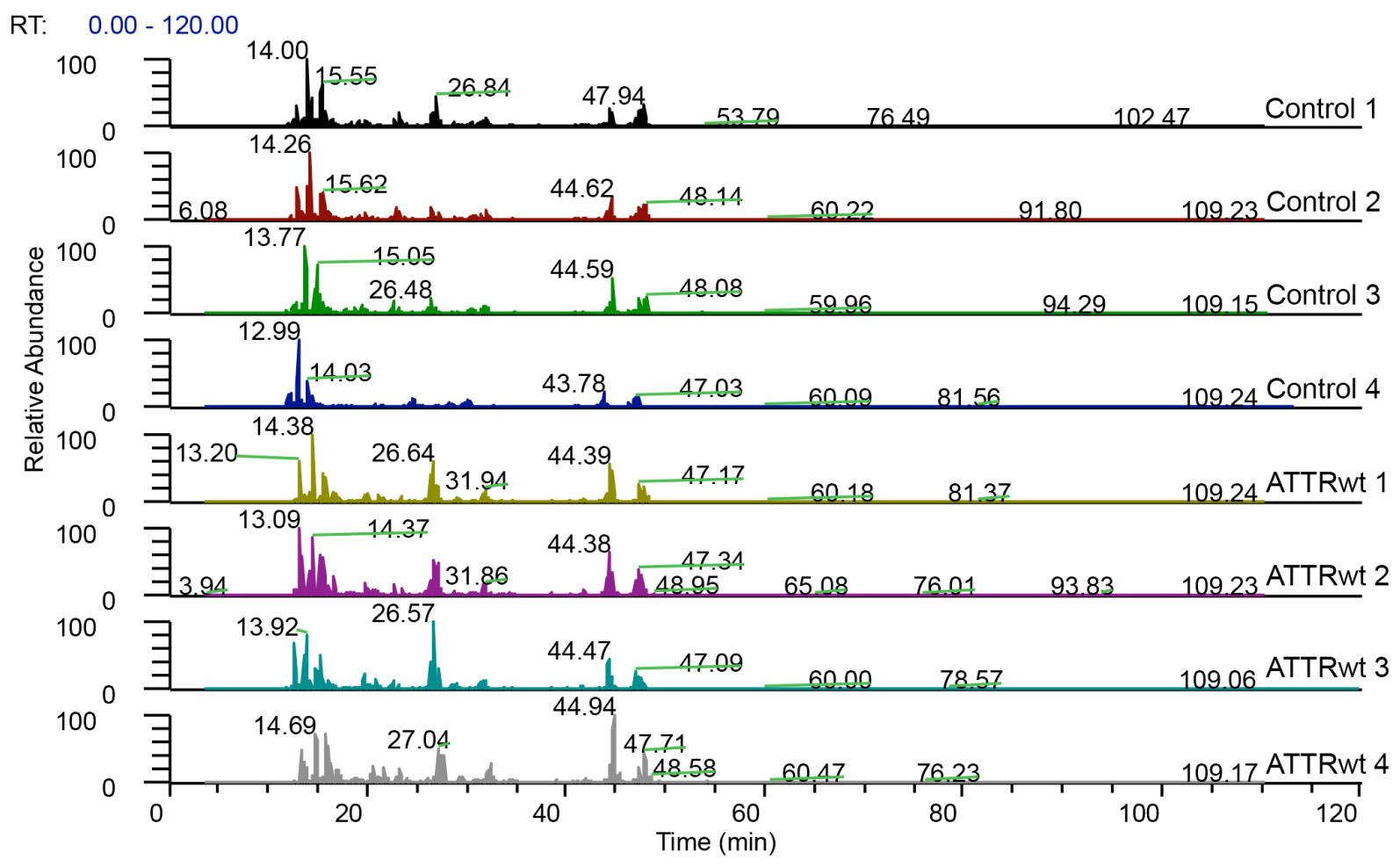

Figure S2. Extracted ion chromatograms for tandem mass spectra of oxonium ions. Fuc, Hex, HexNAc and Hex-HexNAc oxonium ions (signature ions indicating the presence of glycopeptides) were extracted. The chromatograms show a high abundance of glycopeptides as a consequence of incorporating a HILIC-enrichment step during sample preparation. 


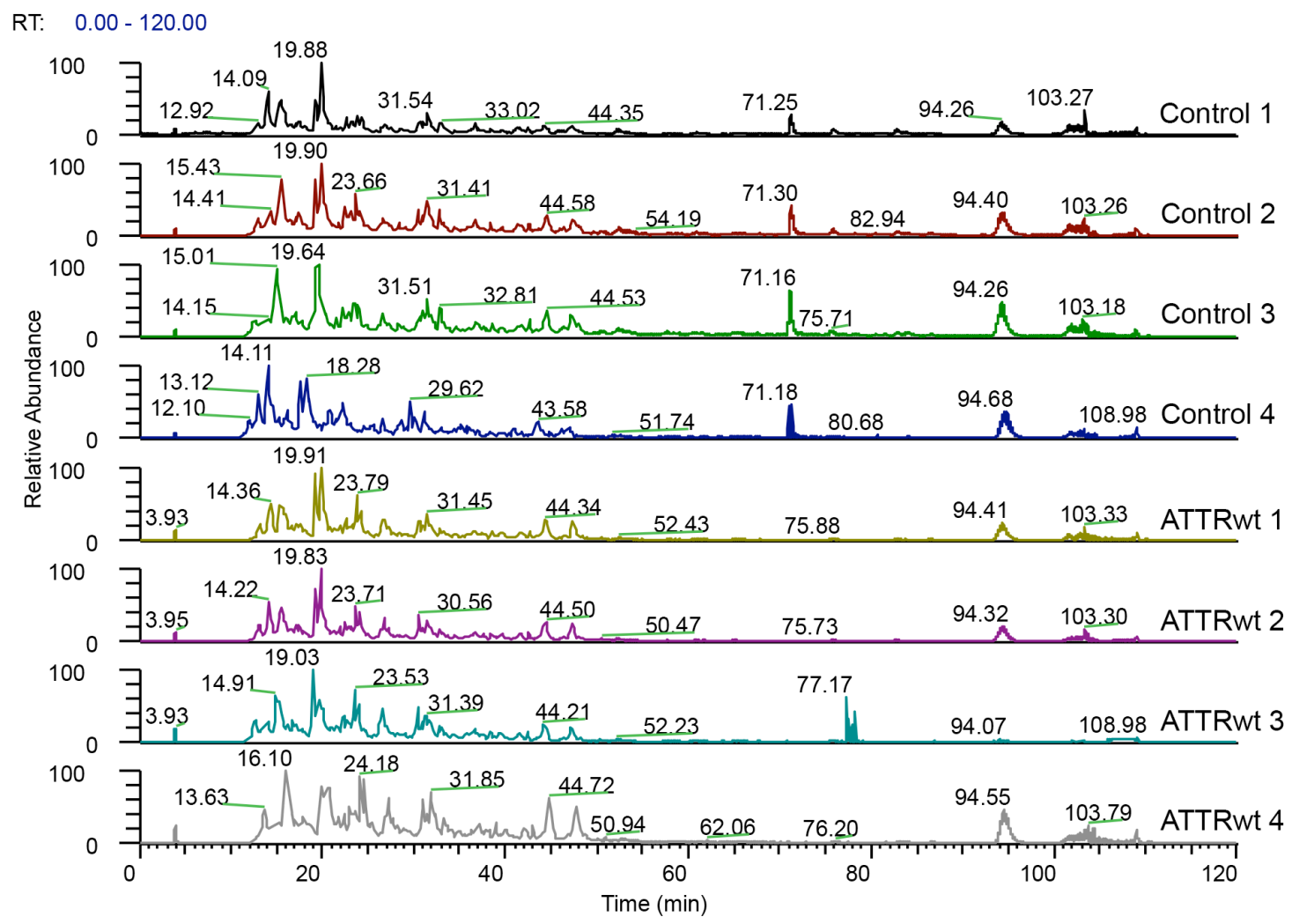

Figure S3. Total Ion Chromatograms 


\begin{tabular}{|c|c|c|c|c|}
\hline & \multirow[b]{3}{*}{$\begin{array}{l}\text { Glycan } \\
\text { structure }\end{array}$} & \multirow{2}{*}{\multicolumn{2}{|c|}{ I T Kanron etal }} & \multirow{3}{*}{ Liang et al. } \\
\hline \multirow[b]{2}{*}{ Site } & & & & \\
\hline & & \multicolumn{2}{|r|}{ Type } & \\
\hline \multirow{9}{*}{$\alpha 64 \mathrm{~N}$} & {$[4 ; 5 ; 0 ; 2]$} & bisialo biantennary & \multirow{9}{*}{$\begin{array}{l}\text { bisialo biantennary } \\
\text { trisialo triantennary } \\
\text { trisialo triantennary with } \\
\text { one fucose }\end{array}$} & bisialo biantennary \\
\hline & {$[5 ; 6 ; 0 ; 3]$} & trisialo triantennary & & trisialo triantennary \\
\hline & {$[5 ; 6 ; 1 ; 3]$} & $\begin{array}{l}\text { trisialo triantennary with } \\
\text { one fucose }\end{array}$ & & $\begin{array}{l}\text { trisialo triantennary with } \\
\text { one fucose }\end{array}$ \\
\hline & {$[6 ; 4 ; 0 ; 4]$} & tetrasialo tetraantennary & & \\
\hline & {$[6 ; 4 ; 2 ; 3]$} & $\begin{array}{l}\text { trisialo tetraantennary } \\
\text { with two fucose }\end{array}$ & & \\
\hline & {$[6 ; 6 ; 0 ; 3]$} & trisialo tetraantennary & & \\
\hline & & & & monosialo biantennary \\
\hline & & & & monosialo triantennary \\
\hline & & & & $\begin{array}{l}\text { bisialo triantennary } \\
\text { bisialo biantennary with } \\
\text { one fucose } \\
\text { bisialo triantennary with } \\
\text { one fucose }\end{array}$ \\
\hline \multirow{4}{*}{$\alpha 81 N$} & {$[6 ; 4 ; 0 ; 4]$} & tetrasialo tetraantennary & & \\
\hline & {$[6 ; 4 ; 2 ; 3]$} & $\begin{array}{l}\text { trisialo tetraantennary } \\
\text { with two fucose }\end{array}$ & & \\
\hline & & & bisialo biantennary & bisialo biantennary \\
\hline & & & $\begin{array}{l}\text { trisialo triantennary } \\
\text { trisialo triantennary with } \\
\text { one fucose }\end{array}$ & $\begin{array}{l}\text { trisialo triantennary } \\
\text { trisialo triantennary with } \\
\text { one fucose }\end{array}$ \\
\hline \multirow{4}{*}{$\alpha 123 \mathrm{~N}$} & {$[4 ; 5 ; 0 ; 2]$} & bisialo biantennary & \multirow[t]{3}{*}{ bisialo biantennary } & \\
\hline & {$[5 ; 5 ; 0 ; 2]$} & bisialo triantennary & & \\
\hline & {$[5 ; 5 ; 1 ; 1]$} & $\begin{array}{l}\text { monosialo triantennary } \\
\text { with one fucose }\end{array}$ & & \\
\hline & & & $\begin{array}{l}\text { trisialo triantennary } \\
\text { trisialo triantennary with } \\
\text { one fucose }\end{array}$ & \\
\hline \multirow{8}{*}{$\beta 269 \mathrm{~N}$} & {$[4 ; 5 ; 0 ; 1]$} & monosialo biantennary & \multirow{8}{*}{$\begin{array}{l}\text { monosialo biantennary } \\
\text { bisialo biantennary }\end{array}$} & monosialo biantennary \\
\hline & {$[4 ; 5 ; 0 ; 2]$} & bisialo biantennary & & bisialo biantennary \\
\hline & {$[4 ; 5 ; 1 ; 2]$} & $\begin{array}{l}\text { bisialo biantennary with } \\
\text { one fucose }\end{array}$ & & $\begin{array}{l}\text { bisialo biantennary with } \\
\text { one fucose }\end{array}$ \\
\hline & {$[5 ; 6 ; 0 ; 2]$} & bisialo triantennary & & bisialo triantennary \\
\hline & {$[5 ; 6 ; 0 ; 3]$} & trisialo triantennary & & trisialo triantennary \\
\hline & & & & monosialo tetraantennary \\
\hline & & & & $\begin{array}{l}\text { monosialo triantennary } \\
\text { monosialo triantennary } \\
\text { with one fucose }\end{array}$ \\
\hline & & & & $\begin{array}{l}\text { bisialo tetraantennary } \\
\text { bisialo triantennary with } \\
\text { one fucose }\end{array}$ \\
\hline
\end{tabular}


trisialo tetraantennary trisialo triantennary with one fucose

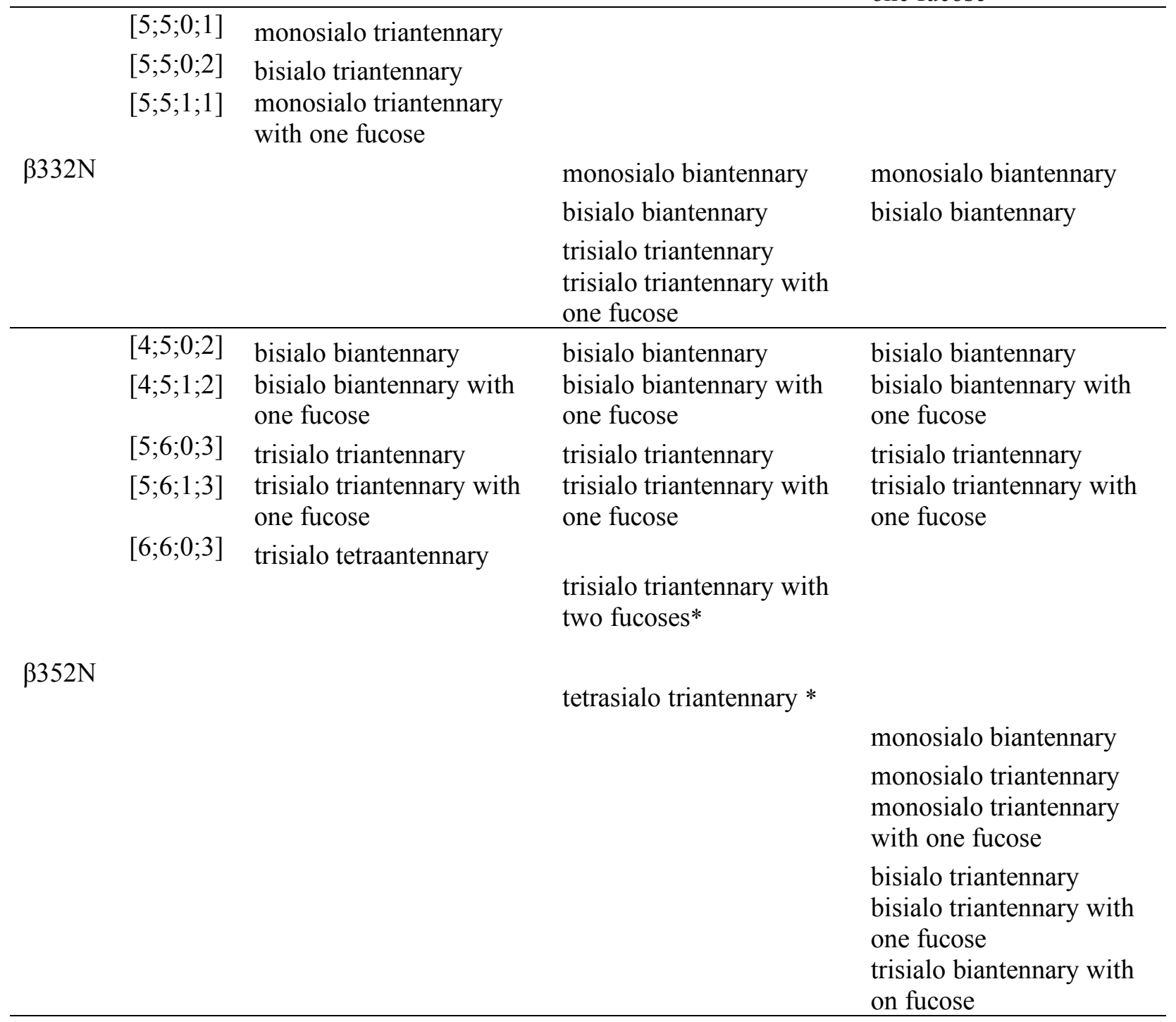

*oligosaccharide compositions are interchangeable.

Table S2. Multi-study comparison of oligosaccharides identified in CLU. Key [HexNAc; Hex; Fuc; Neu5Ac]. 